Published in final edited form as:

Int J Radiat Oncol Biol Phys. 2020 January 01; 106(1): 217-218. doi:10.1016/j.ijrobp.2019.10.044.

\title{
In Regard to Wallner Et Al
}

\section{John Kang ${ }^{1}$, Reid F Thompson ${ }^{2}$, Clifton D Fuller ${ }^{3}$, Kevin A Camphausen ${ }^{4}$, Peter E Gabriel ${ }^{5}$, Charles R Thomas ${ }^{2}$}

${ }^{1}$ Department of Radiation Oncology, University of Rochester Medical Center, Rochester, New York. ${ }^{2}$ Department of Radiation Medicine, Oregon Health \& Science University, Portland, Oregon. ${ }^{3}$ Department of Radiation Oncology, University of Texas MD Anderson Cancer Center, Houston, Texas. ${ }^{4}$ Radiation Oncology Branch, National Cancer Institute, Bethesda, Maryland. ${ }^{5}$ Department of Radiation Oncology, University of Pennsylvania, Philadelphia, Pennsylvania.

\section{To the Editor:}

We read with interest a welcome critique on radiation oncology fellowships by Wallner et al. ${ }^{1}$ We agree with the authors that fellowships for advanced training should be offered for 2 reasons: to focus on opportunities to which residents may have limited exposure during residency — such as protons or brachytherapy — and to provide a path to subspecialty board certification (eg, in Hospice and Palliative Medicine). Clinical Informatics training fulfills both criteria and represents an increasingly important aspect of medical practice.

Competency in informatics is an increasingly marketable skill with multiscale benefits ranging from translational research to institutional quality assessment. A recent American Society for Radiation Oncology survey demonstrated that a large majority of trainees and chairs value additional training in informatics. ${ }^{2}$ Unique among medical specialties is the strong working relationships between radiation oncologists and medical physicists, who are often leading efforts in informatics. This partnership facilitates a natural cancer informatics role for the field of radiation oncology that could elevate its standing within the multidisciplinary oncology team. ${ }^{3}$

The American Board of Preventive Medicine currently offers subspecialty certification in clinical informatics ${ }^{4}$ with a training curriculum formed in cooperation with the American Medical Informatics Association. ${ }^{5}$ Current pathways toward certification include a practice pathway or a 2-year Accreditation Council for Graduate Medical Education-accredited fellowship. The "grandfathered" practice pathway includes either a "time in practice" option or masters/PhD training in biomedical informatics, but it will sunset in 2022. Several of this letter's coauthors are board certified (C.D.F., P.E.G.) or board eligible (R.F.T., J.K.) in clinical informatics via the practice pathway.

To address the emerging need for radiation oncologist-informaticians, the Oregon Health \& Science University, ${ }^{6}$ the University of Texas MD Anderson Cancer Center, ${ }^{7}$ and the National Cancer Institute/Food and Drug Administration ${ }^{8}$ are offering radiation oncology-specific informatics fellowships designed for board eligibility in clinical informatics. These 
fellowships are aimed to position graduates for jobs such as medical director of informatics and/or provide formal informatics certification.

In summary, radiation oncology residents should be aware of a board-certification pathway in clinical informatics and that multiple institutions have created fellowships geared toward training the next generation of oncologist-informaticians.

\section{Acknowledgments/Funding:}

J.K. is supported by an RSNA Research \& Education Foundation Resident Research Grant. C.D.F. is supported by grants from Elekta, NCI, NIDCR, and NIBIB. R.F.T. reports partial employment by Oregon Health \& Science University and is director of its radiation oncology informatics fellowship program. C.D.F. received funding and salary support related to this project from the National Institutes of Health (NIH), including a National Institute of Biomedical Imaging and Bioengineering (NIBIB) Research Education Programs for Residents and Clinical Fellows Grant (R25EB025787-01). C.D.F. received funding and salary support unrelated to this project from the National Institute for Dental and Craniofacial Research Establishing Outcome Measures Award (1R01DE025248/ R56DE025248) and an Academic Industrial Partnership Grant (R01DE028290); National Science Foundation (NSF), Division of Mathematical Sciences, Joint NIH/NSF Initiative on Quantitative Approaches to Biomedical Big Data (QuBBD) Grant (NSF 1557679); the NIH Big Data to Knowledge (BD2K) Program of the National Cancer Institute (NCI) Early Stage Development of Technologies in Biomedical Computing, Informatics, and Big Data Science Award (1R01CA214825); and NCI Early Phase Clinical Trials in Imaging and Image Guided Interventions Program (1R01CA218148). C.D.F. has received direct industry grant support, honoraria, and travel funding from Elekta AB. The contents do not represent the views of the U.S. Department of Veterans Affairs or the U.S. government.

\section{References}

1. Wallner PE, Rosenzweig KE, Vapiwala N. Caveat emptor: Fellow-ship training in radiation oncology; What, but moreimportantly, why? Int J Radiat Oncol Biol Phys2020;106:47-49. [PubMed: 31647970]

2. Mouw KW, Beck TF, Keen JC, Dicker AP. Assessing the training and research environment for genomics, bioinformatics, and immunology in radiation oncology. JCO Clin Can Inform2018;2:1-9.

3. Vapiwala N, Thomas CR, Grover S, et al. Enhancing career paths for tomorrow's radiation oncologists. Int J Radiat Oncol Biol Phys2019;105:52-63. [PubMed: 31128144]

4. Clinical Informatics:American Board of Preventive Medicine.Available at:https://www.theabpm.org/ become-certified/subspecialties/clinical-informatics/. Accessed October 22, 2019.

5. Gardner RM, Overhage JM, Steen EB, et al. Core content for the subspecialty of clinical informatics. J Am Med Inform Assoc2009;16:153-157. [PubMed: 19074296]

6. Fellowship in Clinical Informatics: Radiation Oncology Track: OHSU. Available at:https:// www.ohsu.edu/school-of-medicine/radiation-medicine/fellowship-clinical-informatics-radiationoncology-track. Accessed July 15, 2019.

7. Fuller C, Das P. Fellow and resident radiation oncology intensive training in imaging and informatics to empower research careers(FRONTI2ER). Available at: http://grantome.com/ grant/NIH/R25-EB025787-01. Accessed July 15, 2019.

8. NCI announces oncology data science fellowship. ASTRO: American Society For Radiation Oncology Available at:http://astroblog.weebly.com/1/post/2017/03/nci-announces-oncology-datascience-fellowship.html. Accessed October 22, 2019. 